Tomasz Wasiluk ${ }^{1}$, Kamila Rybinska ${ }^{1}$, Anna Rogowska ${ }^{1}$, Barbara Boczkowska-Radziwon ${ }^{1}$, Piotr Radziwon ${ }^{1,2}$

\title{
Minimally reduced levels of anti-Spike IgG after nine COVID-19 convalescent plasma donations: a case report
}

\author{
Corresponding author: \\ Tomasz Wasiluk \\ Regional Centre for Transfusion Medicine, \\ M. Skłodowskiej-Curie 23, 15-950 \\ Białystok, Poland \\ E-mail: twasiluk@rckik.bialystok.pl
}

Medical Research Journal 2021;

Volume 6, Number 2, 157-160

DOI: 10.5603/MRJ.a2021.0017

Copyright (C) 2021 Via Medica

ISSN 2451-2591

e-ISSN 2451-4101

\begin{abstract}
Despite intensive research, the physiology of the serological response to SARS-CoV-2 infection and its dynamics during the recovery period remain incompletely understood. Regulation of the immune response seems all the more important as it plays a role in both virus clearance during infection and the potential development of long-term resistance to reinfection. A case of convalescent plasma donor is described in whom was observed a prolonged enhanced immune response to infection in the form of a persistently high level of anti-Spike IgG despite subsequent plasma donations. The presented donor experienced COVID-19 interstitial pneumonia, requiring pharmacotherapy in a hospital setting (therapy involving azithromycin, chloroquine and protease inhibitors), which allowed him to achieve remission. The described donor donated plasma nine times during convalescence, each time showing a satisfactory level of anti-Spike lgG. The presented case highlights the multifactorial regulation of the serological response in the course of SARS-CoV-2 infection, which may include the long-term effect of pharmacotherapy, especially in the field of antiretroviral drugs. While the authors are not yet able to clearly define the importance of antiretroviral therapy in regulating the humoral response in COVID-19 patients, it is supposed it may be important in the subsequent antibody production.
\end{abstract}

Key words: antiretroviral, azithromycin, chloroquine, convalescent plasma, COVID-19, SARS-CoV-2

Med Res J 2021; 6 (2): 157-160

\section{Introduction}

The SARS-CoV-2 virus, which is the cause of the global pandemic, is the subject of intense research involving almost the entire world of science. Many aspects of viral biology remain unknown, including the physiology of the serological response to infection and the mechanisms regulating its severity in both asymptomatic and symptomatic (including those developing the COVID-19 disease) patients [1]. Understanding the regulation of the immune response as a reaction to SARSCoV-2 infection seems to be all the more important as it plays a role in both viral clearances during infection and the potential development of long-term immunity to reinfection. While the half-life of anti-SARS-CoV-2 IgG antibodies has been determined to be around 36 days [2], due to the relatively short time of observation of the virus, determining the overall duration of the immune response is not possible yet. It is worth noting that the key elements in the long-term response to infection are ultimately differentiated B-cell lines, known as antibody-secreting cells (ASCs), which determine the humoral immune response and mechanisms that regulate their function [1]. An extremely important issue here seems to be the directing of the B-cell differentiation process towards long-lived or short-lived plasma cells [3], which may determine resistance to reinfection or be no less important in the context of obtaining convalescent plasma for the needs of later transfusions. It should also be assumed that further understanding of the immune response also requires an evaluation of the influence of pharmacotherapy on the modulation of the immune response, both during an ongoing infection and upon extinction of the immune response, understood as the 
recovery phase. A case report of a man with a history of SARS-CoV-2 infection is presented, complicated by COVID-19 interstitial pneumonia, in whom was observed a prolonged enhanced immune response to infection in the form of a persistently high level of anti-SARSCoV-2 antibodies in the IgG class despite subsequent plasma donations.

\section{Case report}

A 52-year-old man came to the facility for a convalescent plasma (CCP) donation having been previously infected with the SARS-CoV-2 virus, complicated by the development of COVID-19 interstitial pneumonia, requiring hospitalization in the Department of Infectious Diseases. The diagnosis was made based on a positive real-time PCR test (nasopharyngeal swab taken on March 30th, 2020) and the presence of characteristic clinical symptoms (fever $>38^{\circ} \mathrm{C}$ and cough, which appeared 3 days after returning from a trip to Georgia). In the laboratory tests performed on admission to the hospital, apart from an increased concentration of C-reactive protein (CRP) (14.25 mg/L, normal range: $0.00-5.00 \mathrm{mg} / \mathrm{L})$ and an increased level of D-Dimer (907.00 ng/mL, normal range: < $500 \mathrm{ng} / \mathrm{mL})$, no significant laboratory abnormalities were found. The complete blood count was within the normal range and the parameters of the white blood cell differential count were as follows: WBC $4.45 \times 10^{3} / \mathrm{mL}$; NEU 2.92 $\times 10^{3} / \mathrm{mL}$; LYMP $1.04 \times 10^{3} / \mathrm{mL}$, MONO $0.46 \times 10^{3} / \mathrm{mL}$, EO $0.01 \times 10^{3} / \mathrm{mL}$; BASO $0.01 \times 10^{3} / \mathrm{mL}$. After the initial stage, during which only febrile episodes and persistent cough were observed, the patient's general condition deteriorated with the development of dyspnoea requiring passive oxygen therapy and interstitial pneumonia, confirmed by a CT scan of the chest. For this reason, treatment with a macrolide antibiotic (azithromycin) was initiated. Due to increasing values of the inflammatory markers (CRP $40.39 \mathrm{mg} / \mathrm{L}$ ), deterioration of the patient's clinical condition and progression of changes in the radiological image (appearance of new lesions with morphology characteristic of COVID-19 pneumonia), antiretroviral therapy (protease inhibitors combination - lopinavir/ritonavir) and chloroquine were added to the pharmacological treatment in an attempt to reverse disease progression. In the following days of hospitalization, improvement in the clinical condition, a decrease in the concentration of inflammatory markers and gradual disappearance of changes in the radiological image were observed. Analysing the clinical course and the results of laboratory and imaging tests, the course of the disease was classified as moderate, based on the developed guidelines for the management of COVID-19 [4]. At discharge from the hospital, the white blood cell differential count presented as follows: WBC $5.62 \times 10^{3} / \mathrm{mL}$; NEU $2.91 \times 10^{3} / \mathrm{mL}$; LYMP $1.92 \times 10^{3} / \mathrm{mL}$, MONO $0.63 \times 10^{3} / \mathrm{mL}$, EO 0.11 $\times 10^{3} / \mathrm{mL}$; BASO $0.03 \times 10^{3} / \mathrm{mL}$. After nearly four weeks of hospitalization, the patient was discharged home in good general condition.

On April 30, 2020, five weeks after obtaining a positive (+) PCR test for SARS-CoV-2 virus RNA and two weeks after the last negative (-) PCR test for SARSCoV-2 virus RNA, the said donor reported to the facility for the first time. Following the qualification procedure, in line with the European Commission's convalescent plasma collection and transfusion program [5], $750 \mathrm{~mL}$ of plasma was collected using an automated plasmapheresis system. No adverse reactions were observed during and after plasmapheresis. In total, nine plasma donations were obtained over four months, at varying intervals, dictated by the work schedule of the donor. In samples taken before each plasma donation, the levels of IgG anti-SARS-CoV-2 antibodies against Spike S1 (Euroimmun, USA), Spike S1/S2 (DiaSorin, Italy) and S-RBD domain (Snibe, China) were determined using the enzyme-linked immunosorbent assay (Spike S1) and chemiluminescent immunoassays (Spike S1/S2 and S-RBD domain). According to the manufacturer's recommendations, the result was considered positive for the following test values: Euroimmun $\mathrm{S} 1 \mathrm{lgG} \geq 1.1$ [S/Co]; Diasorin S1 / S2 lgG $\geq 15[\mathrm{AU} / \mathrm{mL}$; Snibe $S-R B D \operatorname{lgG} \geq 1.0[\mathrm{AU} / \mathrm{mL}]$.

Even though the determination of IgG antibodies against the S1 antigen of the SARS-CoV-2 virus indicates significant dynamics of decrease in the level of antibodies over time, the determination of IgG antibodies against the S1/S2 antigens and S-RBD domain of the virus showed much lower dynamics of decrease. Based on the observations of the described donor, it can be concluded that the time between confirmation of the infection in the PCR-RNA test and the moment when the antibody test is still positive amounts to 157 days.

Importantly, in each of the anti-SARS-CoV-2S1/S2 antibody determinations, the antibody concentration values remained above $80 \mathrm{AU} / \mathrm{mL}$, which, according to the available literature data [6], seems to correlate with the satisfactory level of neutralizing antibodies (nAbs). Also, concerning the anti-S-RBD antibodies, a strong correlation was demonstrated with the titre of neutralizing antibodies [7]. These observations are all the more valuable as conducting tests for the presence of neutralizing antibodies is time-consuming and involves several complicated procedures, such as providing a laboratory with a high microbiological safety class, which limits the implementation of this kind of tests by several institutions. The obtained results are presented in Figure 1. 


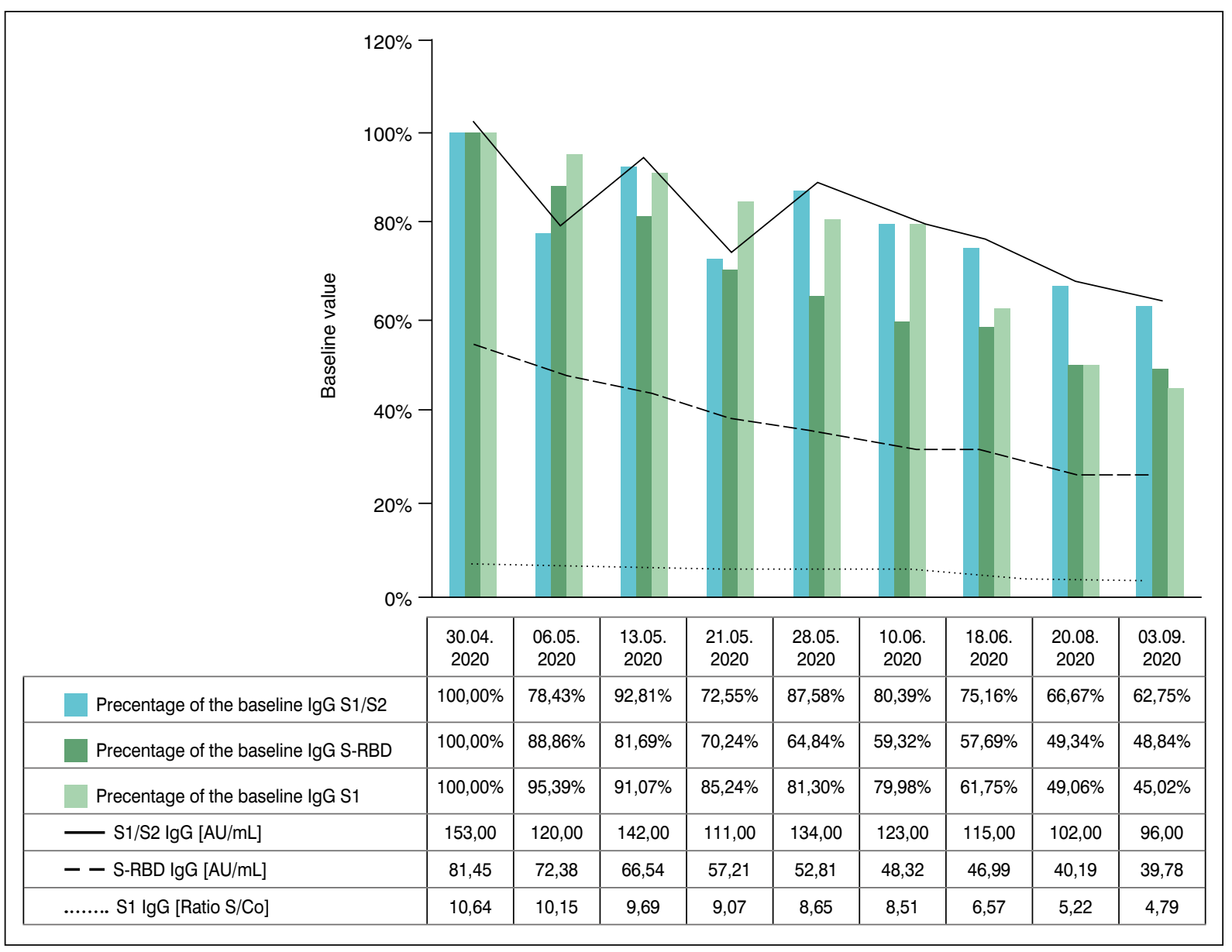

Figure 1. The level of anti-Spike IgG antibodies in subsequent convalescent plasma donations

\section{Discussion}

The presented case suggests that one should not forget about possible immunomodulation related to the applied pharmacotherapy while analysing the humoral response in the course of SARS-CoV-2 infection. Patients whose course of the disease is classified as moderate or severe deserve special attention as it is then that the pharmacotherapy that interferes with the immune system is used to eliminate the virus or stop its intracellular penetration, but also for fear of developing a "cytokine storm". According to the literature, the azithromycin and chloroquine used in this case are associated primarily with suppression of the immune system, including control of the production of pro-inflammatory cytokines such as IL-1 and IL-6 [8]. Direct evidence for the role of drug-induced immunomodulation was provided by studies involving the murine vaccination model, which showed that prior azithromycin therapy led to a significant reduction in the primary humoral response during pneumococcal vaccination [9]. Concerning proteasome inhibitors, the situation is much more complex as they both have a suppressive effect on selected proinflammatory cytokines, being at the same time also characterized by an ability to stimulate T-cell proliferation, which is the most desirable action in their primary indication - HIV infection. Importantly, it has been proved that antiretroviral therapy also significantly affects the proliferation and function of B cells, enhancing the humoral response to both HIV and non-HIV antigens [10]. Although the authors do realize that it is extremely important in obtaining an appropriate immune response during immunization in HIV positive patients [10], they are not yet able to define the significance of this interference with the immune system in the context of COVID-19 patients treated with antiretroviral therapy.

\section{Conclusion}

Although a long-term donor follow-up was conducted, the observations are limited due to single-subject analysis, which makes it difficult to draw firm conclusions. The authors are aware that much more cases are needed to support the presented hypothesis and findings. Nevertheless, the presented report shows the 
multifactorial regulation of the serological response in the course of the SARS-CoV-2 infection, the understanding of which seems to be crucial in the further fight against the ongoing pandemic.

\section{Author contributions: Concept and drafting of the manuscript: TW, KR; critical revision of the manuscript for important intellectual content: $A R$, $B B R, P R$.}

Conflict of interest: The authors declare no conflict of interest.

\section{References}

1. García LF. Immune Response, Inflammation, and the Clinical Spectrum of COVID-19. Front Immunol. 2020; 11: 1441, doi: 10.3389/fimmu.2020.01441, indexed in Pubmed: 32612615.

2. Ibarrondo FJ, Fulcher JA, Goodman-Meza D, et al. Rapid Decay of Anti-SARS-CoV-2 Antibodies in Persons with Mild Covid-19. N Engl J Med. 2020; 383(11): 1085-1087, doi: 10.1056/NEJMc2025179, indexed in Pubmed: 32706954
3. Vaisman-Mentesh A, Dror Y, Tur-Kaspa R, et al. SARS-CoV-2 specific memory B-cells frequency in recovered patient remains stable while antibodies decay over time. , doi: 10.1101/2020.08.23.20179796.

4. Diagnosis and Treatment Protocol for Novel Coronavirus Pneumonia (Trial Version 7). Chin Med J (Engl). 2020; 133(9): 1087-1095, doi: 10.1097/CM9.0000000000000819, indexed in Pubmed: 32358325.

5. An EU programme of COVID-19 convalescent plasma collection and transfusion. Guidance on collection, testing, processing, storage, distribution and monitored use. https://ec.europa.eu/health/sites/health/files/blood_tissues_organs/docs/guidance_plasma_ covid19 en.pdf. (23/06/2020).

6. Bonelli F, Sarasini A, Zierold C, et al. Clinical and Analytical Performance of an Automated Serological Test That Identifies S1/S2-Neutralizing IgG in COVID-19 Patients Semiquantitatively. J Clin Microbiol. 2020; 58(9), doi: 10.1128/JCM.01224-20, indexed in Pubmed: 32580948

7. Coates J. Relationship between Anti-Spike Protein Antibody Titers and SARS-CoV-2 In Vitro Virus Neutralization in Convalescent Plasma. bioRxiv : the preprint server for biology. , doi: 10.1242/prelights.15240.

8. Bleyzac N, Goutelle S, Bourguignon L, et al. Azithromycin for COVID-19: More Than Just an Antimicrobial? Clin Drug Investig. 2020; 40(8): 683-686, doi: 10.1007/s40261-020-00933-3, indexed in Pubmed: 32533455

9. Fernandez AD, Elmore MK, Metzger DW. Azithromycin modulates murine immune responses to pneumococcal conjugate vaccine and inhibits nasal clearance of bacteria. J Infect Dis. 2004; 190(10): 1762-1766, doi: 10.1086/425038, indexed in Pubmed: 15499531.

10. Moir S, Buckner CM, Ho J, et al. B cells in early and chronic HIV infection: evidence for preservation of immune function associated with early initiation of antiretroviral therapy. Blood. 2010; 116(25): 5571-5579, doi: 10.1182/blood-2010-05-285528, indexed in Pubmed: 20837780. 